\title{
The effect of different priming activities performed 24 hours prior to competition
}

\author{
Jacopo Terenzi a, ${ }^{\circledR}$ a and Jeremy Moody $\mathbb{1}$

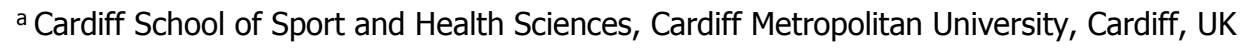 \\ *Corresponding author email: jacopoterenzi@yahoo.com DOI: https://doi.org/10.34256/ijpefs20210 \\ Received: 06-05-2020, Accepted: 27-06-2020
}

\begin{abstract}
The purpose of this study was to examine if performing a lower body resistance training priming activity effects athletic performance completed 24 hours later. University level field hockey players $(N=10)$ completed 1 control (CON) and 2 experimental trials on separate days. A randomised, counter-balanced experimental design with cross-over was used across three conditions. The CON trial consisted of complete abstention from physical activity. For the 'strength' (STR) experimental trial participants performed a parallel back squat exercise for 5 sets of 2 repetitions with 90\% 1RM; while for the 'hypertrophy' (HYP) experimental approach was 3 sets of 10 repetitions with $75 \% 1 \mathrm{RM}$. Subjects attended a testing session 24 hours after each trial, consisting of CMJ, SJ, 22$\mathrm{cm} \mathrm{DJ}, 38-\mathrm{cm}$ DJ and $40 \mathrm{~m}$ sprint. The $5 \mathrm{~m}$ sprint performance was significantly better $(p<0.05)$ for CON group when compared to the HYP group. No other significant differences were highlighted between trials. The results indicate that performing resistance training in-line with STR and HYP the day before competition does not improve athletes' performance. The results also suggest how a lower body STR priming activity can be implemented the day before competition without negatively impact subsequent performance. For the strength and conditioning coach, this may be a useful window to train athletes during the 'in-season' schedule.
\end{abstract}

Keywords: Performance, Potentiation, Priming activity, Strength, Hypertrophy, Power

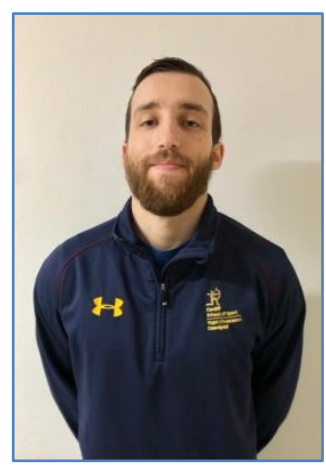

Jacopo Terenzi is the Head Strength and Conditioning Coach at Pesaro Rugby (Italy). He is also the Lead Academy Strength and Conditioning Coach for the Pesaro Rugby Academy (Italy). Other industry experience includes working as a freelance Strength and Conditioning Coach and an internship role at Cardiff Metropolitan University Focus Sports. Jacopo holds an MSc in Strength and Conditioning from Cardiff Metropolitan University and a BSc in Sport and Exercise Science from the University of Urbino (Italy).

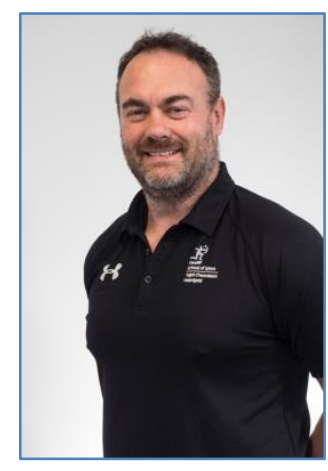

Jeremy Moody is a Senior Lecturer in Strength and Conditioning and Programme Director for the MSc in Strength and Conditioning at Cardiff Met teaching across both the undergraduate and postgraduate schemes in strength and conditioning. Industry based roles have included Regional Lead at the English Institute of
Sport, Performance Manager at UK Athletics, Performance Director for the Great Britain Wheelchair Rugby Association, Performance Director and Chief Operations Officer at Welsh Judo. Jeremy has consulted to Sport Wales, Sport England, UK Sport, various National Governing Bodies and professional sports teams. A member of the UKSCA Board of Directors on a number of occasions and Chairman during $2010-2012$, Jeremy was one of the inaugural groups to establish the current UKSCA accreditation procedure (ASCC).

\section{Introduction}

Getting an athlete in the best physical condition possible, the day of competition, is the primary goal of every strength and conditioning coach. Strength and conditioning professionals wisely manage training and recovery in order to allow their athletes to perform at the highest possible level during competition. The utilisation of pre-conditioning strategies, in the form of appropriate warm-up, passive heat maintenance, post-activation potentiation, remote ischemic preconditioning, hormonal priming and priming activities; it appears to be a useful tool to 
enhance athlete's performance [1]. Kilduff et al., [1] reviewed a number of pre-conditioning strategies, applied on the day of competition, exploring the possible practical use for high intensity sports that includes sprinting, jumping and throwing; proposing a potential timeline of their utilisation in athlete's precompetition routine. Findings suggest that there are many opportunities to enhance the athlete's physical readiness and performance the same day of competition, including the utilisation of resistance training priming activities, a few hours before [1].

There is considerable evidence suggesting that the implementation of a single bout of resistance exercise enhances explosive power measures when performed 3-20 minutes post initial stimulus [2-8]. Current literature has also analysed longer time frames of between four and six hours, with the utilisation of various forms of pre-competition activity [9-13]. Priming activities composed of bouts of sprints with $180^{\circ}$ change of direction at mid-way point or cycle ergometer max-effort protocols, found an enhanced sprinting and power performance in athletes $[9,12]$. Furthermore, several studies have shown that a resistance training session, performed four to six hours before competition enhances strength, power, reactive strength and sprinting performance [9-13]. However, the implementation of priming activities, in the morning, on the day of competition, raises considerable doubt regarding the practicability and the effective use during performance preparation from an applied perspective. The performance of total body resistance training sessions to failure $[9,10]$ or the use of maximal effort sprints a few hours before a game $[9,12]$ is of a doubtful acceptance by coaching staff and players alike, even if power and running velocity may beneficiate from it, later in the day $[9,10,12]$. A logistical issue also emerges if the priming activity needs to be completed a few hours before competition when the match or event is away from home, with the consequent travel needed. It would appear problematic to perform the priming activity session in this case.

Therefore, in a group of university level field hockey players, the aim of this study was to investigate the effects of two different priming activities, 'strength' (STR) and 'hypertrophy' (HYP), on physical performance tests, performed 24 hours later. The purpose is to create a more practical priming activity procedure that can be applied the day before competition, by-passing the issues presented above. Based on the findings of previous research, it was hypothesised that an improvement on physical performances following the STR priming activity would be observed, when compared to the other trials.

\section{Methods}

\subsection{Experimental Approach to the Problem}

The study used a randomised, counterbalanced and cross-over design, with all the subjects completing a testing and familiarisation session followed by 2 experimental (STR; HYP) and 1 control (CON) (no physical activity) trials 24 hours prior to a testing session. The familiarisation session included signing of consent form, the collection of anthropometric data, introduction to study warm-up protocol, a three-repetition maximum (3RM) parallel back squat test and confirmation of familiarisation with the physical testing activities. The familiarisation session and the subsequent trials sessions, including the testing session the day after, were separated by a minimum of 5 days. The timing of priming activities and testing sessions were consistent across the study. A rest day, abstention from caffeine, replication of dietary intake and sleep patterns were requested in the 24 hours before each trial and testing session.

\subsection{Subjects}

The subjects were 10 university level field hockey players aged $20.4 \pm 1.9$ years (body mass 82.4 $\pm 8.8 \mathrm{~kg}$; stature $181.4 \pm 6.4 \mathrm{~cm}$ ). All subjects were required to have completed at least one year of monitored and recorded strength and conditioning training history, with the use of the parallel back squat exercise with intensities up to 3RM. Players volunteered to take part in this study during a period of training (3 resistance training sessions per week) during their off-season. Before participating in the study, all the participants signed an informed consent, approved by the Cardiff Metropolitan University's Ethics Committee. All the subjects were informed of the potential risks associated with the study prior to giving their informed consent by the presentation of a participant information sheet. All subjects declared themselves as injury free and healthy.

\subsection{Procedures}

\subsubsection{Testing and familiarization session}

Physical preparedness for study participation was assessed using the Physical Activity Readiness Questionnaire (PAR-Q). Anthropometric data were taken; stature was recorded using a SECA portable stadiometer (model 321, Vogel \& Halke, Hamburg, 
Germany), while body mass was recorded using a SECA scale (model 770, Vogel \& Halke, Hamburg, Germany). Following collection of anthropometric data, subjects performed a 10-minute predetermined dynamic warm up using a 'RAMP' protocol [14] and a 3RM parallel back squat test. The data taken was used to calculate individual's load for the two priming activities using the Wathen's equation: 1RM= $100 *$ weight/(48.8 $+53.8 * e[-0.075 *$ reps $])$, which has been shown to be the most accurate for the squat exercise $[15,16]$. Following the maximal effort test, the participants were instructed about the physical tests that they would have performed during the research. Participants were provided the opportunity to complete two trials for each test for familiarisation and increase reliability of data.

\subsubsection{Experimental trials}

For the STR and HYP trial, participants arrived in the morning ( 09:00 h) and completed a 10-minute dynamic warm up 'RAMP' protocol [14]; followed by a parallel back squat progressive warmup consisting of 10 repetitions with barbell only $(20 \mathrm{~kg}), 5$ repetitions with $70 \% 1 \mathrm{RM}, 2$ repetitions with $80 \%$ 1RM, 1 repetitions with $90 \% 1 \mathrm{RM}$, with a 2-minute interval inbetween each set for the STR trial and 10 repetitions with barbell only $(20 \mathrm{~kg}), 5$ repetitions with $60 \% 1 \mathrm{RM}$, 5 repetitions with $70 \% 1 \mathrm{RM}$, with a 2-minute interval in-between each set for the HYP trial. After the completion of the warm up the participants commenced the priming activity; STR priming activity consisted of 5 sets of 2 repetitions with $90 \%$ 1RM with a 3-minute interval in-between each set, while, HYP priming activity consisted of 3 sets of 10 repetitions with $75 \% 1$ 1RM with a 90-second interval in-between each set. After the completion of the trial, participants left the gym and were instructed to rest for the remainder of the day. The 3RM parallel back squat test and the two priming activities were performed using 20 kg barbells (Absolute performance Ltd., Cardiff, United Kingdom) and York Fitness plates (York Barbell UK Ltd., Daventry, United Kingdom).

\subsubsection{Control Trial}

For CON trial, participants were instructed to rest for the whole day without doing any physical activity.

\subsubsection{Testing session}

In the morning ( 09:00 $\mathrm{h}$ ) of the day after each trial, participants arrived at the facility and performed a 10-minute dynamic warm up following the 'RAMP' protocol [14] followed by a physical testing battery consisting of body mass measurement, counter movement jump (CMJ), squat jump (SJ), 22-cm drop jump (DJ), 38-cm DJ and a $40 \mathrm{~m}$ sprint, with split times recorded at 5, 10, 20 and 40 meters. CMJ and SJ were performed on a Smartspeed Jump Mat (Smartjump, Fusion Sport, Brisbane, Australia). In the CMJ participants dipped to a self-selected depth before jumping to achieve maximum height. SJ consisted of participants performing a jump from a self-selected isometric squat position held for 3 seconds Two attempts were recorded for both the $\mathrm{CMJ}$ and $\mathrm{SJ}$ with a 2-minute interval in-between repetitions. The highest CMJ height and estimated peak power output (PP), using Sayers' formula $\mathrm{PP}(\mathrm{W})=(51.9) \times$ (jump height in $\mathrm{cm})+(48.9 \mathrm{x}$ body mass -2007$)$, were recorded $[17$, 18], whereas the best SJ height and estimated PP, using Sayers' formula $\operatorname{PP}(\mathrm{W})=(60.7) \times$ (jump height, $\mathrm{cm})+45.3 \times$ body mass -2055$)$, were taken $[18,19]$. The $22-\mathrm{cm}$ and $38-\mathrm{cm}$ DJ were performed from plyometric boxes (Absolute performance Ltd., Cardiff, United Kingdom) on a Smartspeed Jump Mat (Smartjump, Fusion Sport, Brisbane, Australia); two attempts were allowed for each jump, where participants jumped as high as possible with the shortest ground contact time (GCT) as possible. Twominute interval in-between the repetitions were permitted and the best reactive strength index (RSI), calculated from jump height divided by GCT, was recorded. All four types of jump test were performed with participants holding their arms akimbo throughout the jump movement. The $40 \mathrm{~m}$ sprint was recorded with Smartspeed Timing Gates (Smartspeed, Fusion Sport, Brisbane, Australia), with the participants completing the distance in the shortest time. Two attempts were allowed with 2-minute interval inbetween. The fastest time for the $5 \mathrm{~m}, 10 \mathrm{~m}, 20 \mathrm{~m}, 20$ to $40 \mathrm{~m}$ and $40 \mathrm{~m}$ distance were recorded. Participants and tests' order were randomised during each data collection session.

\subsection{Statistical Analyses}

Statistical analyses were carried out using SPSS Statistics software (IBM Inc., USA). Data were reported as mean \pm standard deviation (SD). All data were checked for normality of distribution using Shapiro-Wilk test (above $>0.05$ ) and visual inspection of Histograms, Normal Q-Q plots and Box plots. Difference between performance measures in the various test of the three different trials were analysed using repeated measure ANOVA. Significance in all 
cases was determined with a probability value of $p<$ 0.05 and where appropriate followed by Bonferroni adjusted post hoc test.

Data were also analysed for practical significance using magnitude-based inferences [20]. To compare between-group differences (i.e., CON vs. HYP, CON vs. STR and STR vs. HYP) freely available spreadsheets (http://www.sportsci.org/) were adopted to calculate effect sizes (ES, $90 \% \mathrm{CL}$ ) using the pooled SD of the selected variables of interest [21]. Threshold values for ES statistics were $\leq 0.2$ (trivial), $>0.2$ (small), > 0.6 (moderate), > 1.2 (large), and > 2.0 (very large) [22]. For between-group comparisons uncertainty in the differences were expressed as $90 \%$ $\mathrm{CL}$ and as probabilities that the true (unknown) difference was substantially greater or smaller than the smallest worthwhile change (SWC, 0.2 multiplied by the between-subject standard deviation). Quantitative probabilities of beneficial/better or detrimental/poorer changes/differences were evaluated qualitatively as follows: $<5 \%$, very unlikely; 5-25\%, unlikely; $25-75 \%$, possibly; 75-95\%, likely; 95-99\%, very likely; >99\%, almost certain. If the probabilities of substantially greater and smaller differences were $>5 \%$ the effect was reported as unclear, otherwise the effect was clear and interpreted as the magnitude of the observed value [22].

\section{Results}

Jump performance data for the three different groups and the standardised between-group differences in provided in Table 1 . No significant differences were found between groups. However, there was a possibly small decrease in CMJ height after the HYP condition when compared to the CON condition. Reactive strength data for the three different groups and the standardised between-group differences is possibly provided in Table 2. No significant differences were found between groups. However, there was a small decrease in 22-cm DJ RSI after the HYP trial when compared to the CON trial. Table 3 presents the 'Speed' test data for the three different groups and the standardised between-group differences. The $5 \mathrm{~m}$ sprint performance was significantly better for the CON trial when compared to the HYP trial. There were no significant differences between any other group. However, there was a likely moderate decrease in $5 \mathrm{~m}$ sprint performance after the STR trial when compared to the CON trial. In addition, there was a possibly small decrease in $10 \mathrm{~m}$ and $20 \mathrm{~m}$ sprint performance after the HYP condition when compared to the CON condition.

\section{Discussion}

The current study analysed and compared the effects of different modes of priming activity, performed 24 hours prior, on indicators of athletic performance for university level field hockey players. Priming activities slightly influenced the performance of the athletes, with results which can be usefully applied to the strength and conditioning practice. Despite much research being completed on the effects on performance of resistance training completed within a few hours before different athletic tests [9-13], to the knowledge of the author, no research has explored the effects of resistance training on subsequent athletic performance, investigated through various field tests, performed 24 hours after, and remains conspicuous by its absence.

STR priming activity did not influence positively, the day after performances which contradicts the hypothesis of this study. Analysing the data utilising both Null-Hypothesis Significance Testing and Magnitude Based Inferences analysis, it highlights that the only significant difference was for the $5 \mathrm{~m}$ sprint test, where subjects performing the HYP trial, presented inferior performance compared to the CON group. Similarly, STR trial led to a likely moderate decrease in $5 \mathrm{~m}$ sprint performance, when compared to CON trial. Four variables (i.e., $22 \mathrm{~cm}$ DJ RSI; CMJ height; 10 and $20 \mathrm{~m}$ sprint time) present possible small inferior performances detected in the HYP group if compared to CON group. The findings reveal how neither trial clearly enhanced the athletes' performance 24 hours after, displaying how, utilising the priming activities described in this study, the day before a competition did not provide an additional opportunity in the in-season training cycle, whereby subsequent performance can be enhanced. However, data highlights how a HYP training session can slightly negatively affect the day after performance.

Increased DJ height measures have been reported 6 hours following heavy parallel back squat protocols comprising of 4-6 sets, of 1-4 repetitions, with intensities starting at $80 \% 1$ RM and increasing up to $95 \%$ 1RM [13]. Similarly, rugby players performing a parallel back squat 3RM test, reported significantly enhanced strength and power (i.e., 3RM bench press, 3RM parallel back squat, $\mathrm{CMJ} \mathrm{PP}, 40 \mathrm{~m}$ sprint) measures, 6 hours after [9]. 
Table 1 Descriptive analysis and standardised between-group differences with quantitative and qualitative inferences for the jump performance data scores after 'control', 'hypertrophy' and 'strength' trials

\begin{tabular}{|c|c|c|c|c|c|c|}
\hline & & & & HYP vs. CON & STR vs. CON & STR vs. HYP \\
\hline EXERCISE & $\begin{array}{l}\text { CONTROL } \\
(n=10) \\
\text { mean } \pm \text { SD }\end{array}$ & $\begin{array}{l}\text { HYPERTROPHY } \\
\qquad(n=10) \\
\text { mean } \pm \text { SD }\end{array}$ & $\begin{array}{l}\text { STRENGTH } \\
(n=10) \\
\text { mean } \pm \text { SD }\end{array}$ & $\begin{array}{c}\text { ES }(90 \% \mathrm{CL}) \\
\text { Percentage chances } \\
\text { (+/trivial/-) for HYP - CON } \\
\text { Qualitative inference } \\
\text { P value }\end{array}$ & $\begin{array}{c}\text { ES }(90 \% \mathrm{CL}) \\
\text { Percentage chances } \\
\text { (+/trivial/-) for STR - CON } \\
\text { Qualitative inference } \\
\text { P value }\end{array}$ & $\begin{array}{c}\text { ES }(90 \% \mathrm{CL}) \\
\text { Percentage chances } \\
\text { (+/trivial/-) for STR - HYP } \\
\text { Qualitative inference } \\
\text { P value }\end{array}$ \\
\hline $\begin{array}{c}\text { CMJ } \\
\text { HEIGHT } \\
(\mathrm{cm})\end{array}$ & $36.03 \pm 5.20$ & $34.38 \pm 6.05$ & $\begin{array}{c}34.80 \pm \\
7.51\end{array}$ & $\begin{array}{c}-0.27(-0.49 ;-0.05) \\
0 / 29 / 71 \\
\text { Possibly } \\
P=0.159\end{array}$ & $\begin{array}{c}-0.17(-0.47 \\
0.12) \\
2 / 54 / 44 \\
\text { Possibly } \\
P=0.910\end{array}$ & $\begin{array}{c}0.06(-0.22 ; 0.33) \\
18 / 76 / 6 \\
\text { Unclear } \\
P=1.000\end{array}$ \\
\hline $\begin{array}{l}\text { CMJ PP } \\
\text { (W) }\end{array}$ & $\begin{array}{c}3916.77 \pm \\
508.87\end{array}$ & $\begin{array}{c}3841.61 \pm \\
464.03\end{array}$ & $\begin{array}{c}3856.30 \pm \\
449.28\end{array}$ & $\begin{array}{c}-0.14(-0.30 ; 0.02) \\
0 / 74 / 25 \\
\text { Possibly } \\
P=0.339\end{array}$ & $\begin{array}{c}-0.12(-0.33 ; 0.10) \\
1 / 75 / 24 \\
\text { Possibly trivial } \\
P=1.000\end{array}$ & $\begin{array}{c}0.03(-0.19 ; 0.25) \\
9 / 86 / 4 \\
\text { Likely trivial } \\
P=1.000\end{array}$ \\
\hline $\begin{array}{l}\text { SJ HEIGHT } \\
(\mathrm{cm})\end{array}$ & $33.20 \pm 6.56$ & $33.48 \pm 5.72$ & $\begin{array}{c}33.16 \pm \\
7.04\end{array}$ & $\begin{array}{c}0.04(-0.21 ; 0.30) \\
14 / 80 / 6 \\
\text { Unclear } \\
\mathrm{P}=1.000\end{array}$ & $\begin{array}{c}-0.01(-0.14 ; 0.13) \\
1 / 98 / 1 \\
\text { Very likely trivial } \\
P=1.000\end{array}$ & $\begin{array}{c}-0.05(-0.34 ; 0.25) \\
8 / 73 / 18 \\
\text { Unclear } \\
P=1.000\end{array}$ \\
\hline SJ PP (W) & $\begin{array}{c}3715.79 \pm \\
508.36\end{array}$ & $\begin{array}{c}3742.30 \pm \\
366.53\end{array}$ & $\begin{array}{c}3716.54 \pm \\
512.39\end{array}$ & $\begin{array}{c}0.05(-0.30 ; 0.02) \\
13 / 84 / 3 \\
\text { Likely trivial } \\
\mathrm{P}=1.000\end{array}$ & $\begin{array}{l}0.00(-0.33 ; 0.10) \\
\quad 1 / 99 / 0 \\
\text { Very likely trivial } \\
\quad \mathrm{P}=1.000\end{array}$ & $\begin{array}{c}-0.05(-0.31 ; 0.20) \\
5 / 79 / 16 \\
\text { Unclear } \\
P=1.000\end{array}$ \\
\hline
\end{tabular}

Note. $\mathrm{n}$, sample size; $\mathrm{ES}$, effect size, standardized differences; $90 \% \mathrm{CL}, 90 \%$ confidence limits. Qualitative inferences are $\leq 0.2$ (trivial), $>0.2$ (small), > 0.6 (moderate), > 1.2 (large), and > 2.0 (very large): <5\%, very unlikely; 5-25\%, unlikely; 25-75\%, possibly; 75-95\%, likely; $95-99 \%$, very likely; $>99 \%$, almost certain. $*$ significant difference between groups. 
Table 2 Descriptive analysis and standardised between-group differences with quantitative and qualitative inferences for the Reactive strength index scores after 'control', 'hypertrophy' and 'strength' trials

\begin{tabular}{|c|c|c|c|c|c|c|}
\hline & & & & HYP vs. CON & STR vs. CON & STR vs. HYP \\
\hline EXERCISE & $\begin{array}{l}\text { CONTROL } \\
(n=10) \\
\text { mean } \pm \text { SD }\end{array}$ & $\begin{array}{l}\text { HYPERTROPHY } \\
(n=10) \\
\text { mean } \pm \text { SD }\end{array}$ & $\begin{array}{l}\text { STRENGTH } \\
(n=10) \\
\text { mean } \pm \text { SD }\end{array}$ & $\begin{array}{c}\text { ES }(90 \% \mathrm{CL}) \\
\text { Percentage chances } \\
\text { (+/trivial/-) for HYP - } \\
\text { CON } \\
\text { Qualitative inference } \\
\text { P value }\end{array}$ & $\begin{array}{c}\text { ES }(90 \% \mathrm{CL}) \\
\text { Percentage chances } \\
\text { (+/trivial/-) for STR - } \\
\text { CON } \\
\text { Qualitative inference } \\
\text { P value }\end{array}$ & $\begin{array}{c}\text { ES }(90 \% \mathrm{CL}) \\
\text { Percentage chances } \\
\text { (+/trivial/-) for STR - HYP } \\
\text { Qualitative inference } \\
\text { P value }\end{array}$ \\
\hline $\begin{array}{c}22 \mathrm{~cm} \mathrm{DJ} \\
\text { RSI }\end{array}$ & $1.50 \pm 0.45$ & $1.39 \pm 0.45$ & $1.46 \pm 0.56$ & $\begin{array}{c}-0.23(-0.46 ; 0.00) \\
0 / 40 / 59 \\
\text { Possibly } \\
\mathrm{P}=0.292\end{array}$ & $\begin{array}{c}-0.08(-0.40 ; 0.24) \\
7 / 67 / 26 \\
\text { Unclear } \\
P=1.000\end{array}$ & $\begin{array}{c}0.12(-0.15 ; 0.39) \\
30 / 67 / 3 \\
\text { Possibly } \\
P=1.000\end{array}$ \\
\hline $\begin{array}{c}38 \mathrm{~cm} \mathrm{DJ} \\
\text { RSI }\end{array}$ & $1.52 \pm 0.48$ & $1.49 \pm 0.50$ & $1.54 \pm 0.56$ & $\begin{array}{c}-0.06(-0.41 ; 0.28) \\
10 / 66 / 24 \\
\text { Unclear } \\
P=1.000\end{array}$ & $\begin{array}{c}0.03(-0.28 ; 0.35) \\
18 / 72 / 11 \\
\text { Unclear } \\
P=1.000\end{array}$ & $\begin{array}{c}0.09(-0.22 ; 0.40) \\
26 / 68 / 6 \\
\text { Unclear } \\
\mathrm{P}=1.000\end{array}$ \\
\hline
\end{tabular}

Note. $\mathrm{n}$, sample size; $\mathrm{ES}$, effect size, standardized differences; $90 \% \mathrm{CL}, 90 \%$ confidence limits. Qualitative inferences are $\leq 0.2$ (trivial), > 0.2 (small), > 0.6 (moderate), > 1.2 (large), and > 2.0 (very large): <5\%, very unlikely; 5-25\%, unlikely; 25-75\%, possibly; 75-95\%, likely; $95-99 \%$, very likely; $>99 \%$, almost certain. * significant difference between groups. 
Table 3 Descriptive analysis and standardised between-group differences with quantitative and qualitative inferences for the Speed exercises scores after 'control', 'hypertrophy' and 'strength' trials

\begin{tabular}{|c|c|c|c|c|c|c|}
\hline & & & & HYP vs. CON & STR vs. CON & STR vs. HYP \\
\hline EXERCISE & $\begin{array}{l}\text { CONTROL } \\
(n=10) \\
\text { mean } \pm \text { SD }\end{array}$ & $\begin{array}{l}\text { HYPERTROPHY } \\
\qquad(n=10) \\
\text { mean } \pm \text { SD }\end{array}$ & $\begin{array}{l}\text { STRENGTH } \\
(n=10) \\
\text { mean } \pm \text { SD }\end{array}$ & $\begin{array}{c}\text { ES }(90 \% \mathrm{CL}) \\
\text { Percentage chances } \\
\text { (+/trivial/-) for HYP - CON } \\
\text { Qualitative inference } \\
\text { P value }\end{array}$ & $\begin{array}{c}\text { ES }(90 \% \mathrm{CL}) \\
\text { Percentage chances } \\
\text { (+/trivial/-) for STR - CON } \\
\text { Qualitative inference } \\
\text { P value }\end{array}$ & $\begin{array}{c}\text { ES }(90 \% \mathrm{CL}) \\
\text { Percentage chances } \\
\text { (+/trivial/-) for STR - HYP } \\
\text { Qualitative inference } \\
\text { P value }\end{array}$ \\
\hline $\begin{array}{c}5 \mathrm{~m} \text { SPRINT } \\
\text { (s) }\end{array}$ & $1.00 \pm 0.02$ & $1.03 \pm 0.04$ & $1.03 \pm 0.04$ & $\begin{array}{c}0.73(0.28 ; 1.18) \\
97 / 3 / 0 \\
\text { Very likely } \\
P=0.047^{*}\end{array}$ & $\begin{array}{c}0.69(0.11 ; 1.28) \\
92 / 7 / 1 \\
\text { Likely } \\
\mathrm{P}=0.172\end{array}$ & $\begin{array}{c}-0.07(-0.57 ; 0.44) \\
18 / 50 / 32 \\
\text { Unclear } \\
P=1.000\end{array}$ \\
\hline $\begin{array}{c}10 \mathrm{~m} \\
\text { SPRINT (s) }\end{array}$ & $1.77 \pm 0.04$ & $1.79 \pm 0.07$ & $1.78 \pm 0.07$ & $\begin{array}{c}0.25(-0.13 ; 0.63) \\
59 / 38 / 3 \\
\text { Possibly } \\
P=0.784\end{array}$ & $\begin{array}{c}0.14(-0.31 ; 0.59) \\
41 / 49 / 10 \\
\text { Unclear } \\
P=1.000\end{array}$ & $\begin{array}{c}-0.09(-0.42 ; 0.24) \\
7 / 65 / 28 \\
\text { Unclear } \\
P=1.000\end{array}$ \\
\hline $\begin{array}{c}20 \mathrm{~m} \\
\text { SPRINT (s) }\end{array}$ & $3.08 \pm 0.09$ & $3.11 \pm 0.12$ & $3.10 \pm 0.11$ & $\begin{array}{c}0.25(0.02 ; 0.48) \\
65 / 34 / 0 \\
\text { Possibly } \\
P=0.233\end{array}$ & $\begin{array}{c}0.14(-0.19 ; 0.48) \\
38 / 57 / 5 \\
\text { Possibly } \\
P=1.000\end{array}$ & $\begin{array}{c}-0.10(-0.41 ; 0.21) \\
5 / 66 / 29 \\
\text { Unclear } \\
P=1.000\end{array}$ \\
\hline $\begin{array}{c}20-40 \mathrm{~m} \\
\text { SPRINT (s) }\end{array}$ & $2.47 \pm 0.10$ & $2.49 \pm 0.15$ & $2.46 \pm 0.13$ & $\begin{array}{c}0.08(-0.27 ; 0.43) \\
27 / 64 / 9 \\
\text { Unclear } \\
P=1.000\end{array}$ & $\begin{array}{c}-0.08(-0.38 ; 0.22) \\
6 / 70 / 24 \\
\text { Unclear } \\
P=1.000\end{array}$ & $\begin{array}{c}-0.14(-0.33 ; 0.06) \\
1 / 72 / 28 \\
\text { Possibly } \\
P=0.672\end{array}$ \\
\hline $\begin{array}{c}40 \mathrm{~m} \\
\text { SPRINT (s) }\end{array}$ & $5.56 \pm 0.18$ & $5.60 \pm 0.27$ & $5.56 \pm 0.24$ & $\begin{array}{c}0.16(-0.14 ; 0.45) \\
40 / 57 / 3 \\
\text { Possibly } \\
P=1.000\end{array}$ & $\begin{array}{c}0.00(-0.02 ; 0.03) \\
0 / 100 / 0 \\
\text { Most likely trivial } \\
\mathrm{P}=1.000\end{array}$ & $\begin{array}{c}-0.01(-0.03 ; 0.01) \\
0 / 100 / 0 \\
\text { Most likely trivial } \\
\mathrm{P}=1.000\end{array}$ \\
\hline
\end{tabular}

Note. $\mathrm{n}$, sample size; ES, effect size, standardized differences; $90 \% \mathrm{CL}, 90 \%$ confidence limits. Qualitative inferences are $\leq 0.2$ (trivial), $>0.2$ (small), $>0.6$ (moderate), > 1.2 (large), and > 2.0 (very large): <5\%, very unlikely; 5-25\%, unlikely; 25-75\%, possibly; $75-95 \%$, likely; $95-99 \%$, very likely; $>99 \%$, almost certain. $*$ significant difference between groups. 
Because this study examined a similar resistance training session (i.e., 5 sets of 2 repetitions with $90 \% 1 \mathrm{RM}$ ) to those utilised in the previously cited studies $[9,13]$, but performed 24 hours before the testing session; it can be hypothesised that the mechanisms which could be associated with the improved performance, such as hormonal change and positive psychological mood outcomes, could have disappeared over a timeframe longer than 6 hours [9, 13 , 23]. One other study has confirmed that a resistance training session, in the form of parallel back squat up to $1 \mathrm{RM}$ and power clean up to 4RM, does improve backwards overhead shot throw (BOST) in experienced shot-putters 6 hours later [10]. However, data also reported a decrease in CMJ performance following the training session [10]. Authors suggested that the decrease in jumping performance in their study could be related to the specificity of the lifting exercises utilised [10].

A recent study by Russell et al., [12] investigated different types of priming activities (i.e., sprinting; cycling; resistance training) implemented in the morning by high level French rugby players, detecting how afternoon jumping performance (i.e., $\mathrm{CMJ}$ ) did improve after the sprinting (i.e., six $40 \mathrm{~m}$ sprints with $180^{\circ}$ change of direction) and cycling (i.e., six $6 \mathrm{~s}$ cycle ergometer sprints) protocols and not the resistance training (i.e., bench press 5 sets of 10 repetitions with $75 \% 1 \mathrm{RM}$ ) one. It could be argued that the resistance training priming activity not influencing the afternoon test may be due to the noninvolvement of the lower body. Another study utilising auxotonic resistance training exercises, found an increase in bench throws performance, coupled with a decrease in squat jump performance, after one hour and 45 minutes from implementing a priming activity composed of a parallel back squat with bands as a resistance (4 sets of 3 repetition with $66,8 \pm 5.3 \mathrm{~kg}$ ) and a bench press with bands as a resistance ( 4 sets of 3 repetitions with $66,8 \pm 5.3 \mathrm{~kg}$ ) [11]. Authors hypothesised that due to subjects' higher strength level on the lower body compared to the upper body, external load during the band parallel back squat was not sufficient enough to elicit a potentiating effect [11].

Multiple studies analysed the effects of previous field or resistance training activity on testosterone salivary concentrations and its circadian rhythm [9, 12, 24-28]. Higher testosterone concentrations have been correlated with increased levels of athletic performance [24, 26, 29]. Especially, higher performances on the parallel back squat exercise and sprinting appears to be correlated with high concentration of salivary testosterone in elite athletes [25]. Pre-game testosterone levels have also confirmed relationships with match outcomes in rugby union [27], and increased three repetition maximum strength was recorded after an increase in testosterone levels due to a visual stimulation [24].

Short-term study (i.e., 6 hours) on salivary testosterone concentrations demonstrated how heavy (i.e., up to $100 \%$ of $3 R M$ ) bench press and back squat exercises positively attenuated testosterone circadian decline [9]. A similar study, reported that a weight (i.e., bench press 5 sets of 10 repetitions with $75 \%$ 1RM load) priming activity performed in the morning have a positive effect $(+21 \pm 23 \mathrm{pg} \cdot \mathrm{ml}-1,+17 \pm 18 \%$, $\mathrm{P}=0.002$ ) on testosterone decline in the afternoon compared to complete resting group [12]. Kraemer et al., [28] investigated circadian rhythms of serum testosterone concentrations in men, after a resistance training workout and the day after; comparing them to the circadian rhythms following complete rest. The protocol utilised in the current research is clearly in-line to the one employed in this study. Interestingly, similarity was found in the response pattern of salivary testosterone between both heavy resistance and resting condition. Heavy resistance exercise did not cause a significant increase in the magnitude of testosterone secretion following the bout of exercises, but neither increased the testosterone levels throughout the following day $[28,29]$.

The novel finding of the study was that performing a STR (i.e., 5 sets of 2 repetitions with $90 \% 1 \mathrm{RM}$ of parallel back squat) resistance training session did not significantly decline power, reactive strength and sprinting performance, when performed 24 hours prior, if compared to a CON group who did not perform any physical activity. The only decrease in performance following the STR priming activity was likely moderate in the $5 \mathrm{~m}$ sprint, when compared to CON group (ES of $1.03 \pm 0.04$ and $1.00 \pm 0.02$, respectively). A possible limitation was the non-elite level of the athletes tested, combined with a small sample size. Influences of familiarisation with the power, reactive strength and sprint tests were minimised by the participants' previous experience with these tests during the strength and conditioning training, combined with subjects' practice of the different tests during the familiarisation session. However, the reader should consider that the aim of this research was to investigate if a determined type of resistance training session could enhance the day after 
(i.e., 24 hours) performance on different field tests. Further studies should validate these findings, testing a larger number of subjects.

\section{Conclusions}

The study reports novel data concerning the efficacy of different modes of resistance training priming activities on multiple athletic performances 24 hours later. The findings of this study indicate how both STR and HYP priming activities did not increase on-field performance measures 24 hours later. However, the lower body 'strength' resistance training session did not decrease the athletes' tests outcome. This peculiar finding suggests that for university level field hockey players, performing a resistance training workout with high intensity (i.e., 90\% 1RM) and low volume ( 5 sets of 2 repetitions), with a 3-minute interval in-between each set, the day before a competition, could represent an opportunity to perform resistance training, without negatively affecting competition 24 hours later. The findings of this study may assist strength and conditioning coaches in implementing similar resistance training sessions during the in-season part of the annual cycle, providing an additional window of training opportunity.

\section{References}

[1] L.P. Kilduff, C.V. Finn, J.S. Baker, C.J. Cook, and D.J. West, Preconditioning strategies to enhance physical performance on the day of competition, International Journal of Sports Physiology and Performance, 8 (2013) 677-681.

https://doi.org/10.1123/ijspp.8.6.677

[2] P. Aagaard, E.B. Simonsen, J.L. Andersen, P. Magnusson, and P. Dyhre-Poulsen, Increased rate of force development and neural drive of human skeletal muscle following resistance training, Journal of Applied Physiology, 93 (2002) 1318-1326.

https://doi.org/10.1152/japplphysiol.00283.2002

[3] V. Gourgoulis, N. Aggeloussis, P. Kasimatis, G. Mavromatis, and A. Garas, Effect of a submaximal half-squats warm-up program on vertical jumping ability, Journal of Strength and Conditioning Research, 17 (2003) 342-344.

https://doi.org/10.1519/1533-

4287(2003)017\%3C0342:eoashw\%3E2.0.c0;2

[4] A. Güllich, and D. Schmidtbleicher, Short-term increase of explosive strength through maximal arbitrary contractions: The relation of neurolan involution [German]. Leistungssport 27: 1997.
[5] M.J. Matthews, H.P. Matthews, and B. Snook, The acute effects of a resistance training warmup on sprint performance, Research in Sports Medicine, 12 (2004) 151-159. https://doi.org/10.1080/15438620490460503

[6] D.G. Sale, Postactivation potentiation: Role in human performance, Exercise and Sport Sciences Reviews, 30 (2002) 138-143.

https://doi.org/10.1097/00003677-200207000$\underline{00008}$

[7] A.J. Sargent, and P. Dolan, Effect of prior exercise on maximal short-term power output in humans, Journal of Applied Physiology, 63 (1987) 1475-1480.

https://doi.org/10.1152/jappl.1987.63.4.1475

[8] W.B. Young, A. Jenner, and K. Griffiths, Acute enhancement of power performance from heavy load squats, Journal of Strength and Conditioning Research, 12 (1998) 82-84.

http://dx.doi.org/10.1519/15334287(1998)012<0082:AEOPPF>2.3.CO;2

[9] C.J. Cook, L.P. Kilduff, B.T. Crewther, M. Beaven, and D.J. West, Morning based strength training improves afternoon physical performance in rugby union players, Journal of Science and Medicine in Sport, 17 (2014) 317-321.

https://doi.org/10.1016/j.jsams.2013.04.016

[10] L.G. Ekstrand, C.L. Battaglini, R.G. McMurray, and E.W. Shields, Assessing explosive power production using the backward overhead shot throw and the effects of morning resistance exercise on afternoon performance, Journal of Strength and Conditioning Research, 27 (2013) 101-106.

https://doi.org/10.1519/jsc.0b013e3182510886

[11] B.R. Mason, C.K. Argus, B. Norcott, and N.B. Ball, Resistance Training Priming Activity Improves Upper-Body Power Output in Rugby Players: Implications for Game Day Performance, Journal of Strength and Conditioning Research, 31 (2017) 913-920.

https://doi.org/10.1519/jsc.0000000000001552

[12] M. Russell, A. King, R.M. Bracken, C.J. Cook, T. Giroud, and L.P. Kilduff, A Comparison of Different Modes of Morning Priming Exercise on Afternoon Performance, International Journal of Sports Physiology and Performance, 11 (2016) 763-767.

https://doi.org/10.1123/ijspp.2015-0508

[13] E. Saez Saez de Villarreal, J.J. González-Badillo, and M. Izquierdo, Optimal warm-up stimuli of muscle activation to enhance short and long- 
term acute jumping performance, European Journal of Applied Physiology, 100 (2007) 393401.

https://doi.org/10.1007/s00421-007-0440-9

[14] I. Jeffreys, Warm up revisited- the 'RAMP' method of optimising performance preparation, Professional Strength and Conditioning 6 (2007) 15-19.

[15] D.A. Lesuer, J.H. McCormick, J.L. Mayhew, R.L. Wasserstein, and D.M. Arnold, The Accuracy of seven predictions for estimating 1-RM performance in the bench press, squat, and deadlift,Journal of Strength \& Conditioning Research 11 (1997) 211-213.

https://doi.org/10.1519/00124278-19971100000001

[16] F.J. Naclerio, A. Jiménez, B.A. Alvar, and M.D. Peterson, Assessing strength and power in resistance training, Journal of Human Sport and Exercise 4 (2009) 100-113. https://doi.org/10.4100/jhse.2009.42.04

[17] M.J. Duncan, M. Lyons, and A.M. Nevill, Evaluation of Peak Power Prediction Equations in Male Basketball Players', Journal of Strength and Conditioning Research, 22 (2008) 1379-1381. https://doi.org/10.1519/jsc.0b013e31816a6337

[18] S.P. Sayers, D.V. Harackiewicz, E.A. Harman, P.N. Frykman, and M.T. Rosenstein, Crossvalidation of three jump power equations, Medicine and Science in Sports and Exercise 31 (1999) 572-577.

https://doi.org/10.1097/00005768-199904000$\underline{00013}$

[19] J.M. Carlock, S.L. Smith, M.J. Hartman, R.T. Morris, D.A. Ciroslan, K.C. Pierce, R.U. Newton, E.A. Harman, W.A. Sands, and M.H. Stone, The relationship between vertical jump power estimates and weightlifting ability: a field-test approach, Journal of Strength \& Conditioning Research, 18 (2004) 534-539.

https://doi.org/10.1519/r-13213.1

[20] A.M. Batterham, and W.G. Hopkins, Making meaningful inferences about magnitudes, International Journal of Sports Physiology and Performance, 1 (2006) 50-57.

https://doi.org/10.1123/ijspp.1.1.50

[21] W.G. Hopkins, Spreadsheets for analysis of controlled trials, with adjustment for a subject characteristic, Sportscience 10 (2006) 46-50.

[22] W.G. Hopkins, S.W. Marshall, A.M. Batterham, and J. Hanin, Progressive statistics for studies in sports medicine and exercise science, Medicine \& Science in Sports \& Exercise, 41 (2009) 3-13. https://doi.org/10.1249/mss.0b013e31818cb278

[23] A.C. Fry, M.H. Stone, J.T. Thrush, and S.J. Fleck, Precompetition training sessions enhance competitive performance of high-anxiety junior weightlifters, Journal of Strength and Conditioning Research, 9 (1995) 37-42.

http://dx.doi.org/10.1519/00124278-199502000$\underline{00008}$

[24] C.J. Cook, and B.T. Crewther, Changes in salivary testosterone concentrations and subsequent voluntary squat performance following the presentation of short video clips, Hormones and Behavior, 61 (2012) 17-22.

https://doi.org/10.1016/j.yhbeh.2011.09.006

[25] B.T. Crewther, C.J. Cook, C.M. Gaviglio, L.P. Kilduff, and S. Drawer, Baseline strength can influence the ability of salivary free testosterone to predict squat and sprinting performance, Journal of Strength and Conditioning Research, 26 (2012) 261-268.

https://doi.org/10.1519/jsc.0b013e3182185158

[26] B.T. Crewther, L.P. Kilduff, C.J. Cook, D.J. Cunningham, P. Bunce, R.M. Bracken, and C.M. Gaviglio, Relationships between salivary free testosterone and the expression of force and power in elite athletes, Journal of Sports Medicine and Physical Fitness 52 (2012) 221227.

[27] C.M. Gaviglio, B.T. Crewther, L.P. Kilduff, K.A. Stokes, and C.J. Cook, Relationship between pregame concentrations of free testosterone and outcome in rugby union, International Journal of Sports Physiology and Performance, 9 (2014) 324-331.

\section{https://doi.org/10.1123/ijspp.2013-0106}

[28] W.J. Kraemer, C.C. Loebel, J.S. Volek, N.A. Ratamess, R.U. Newton, R.B. Wickham, L.A. Gotshalk, N.D. Duncan, S.A. Mazzetti, A.L. Gómez, M.R. Rubin, B.C. Nindl, and K. Häkkinen, The effect of heavy resistance exercise on the circadian rhythm of salivary testosterone in men, European Journal of Applied Physiology 84 (2001) 13-18. https://doi.org/10.1007/s004210000322

[29] B.T. Crewther, T. Lowe, R.P. Weatherby, N. Gill, and J. Keogh, Neuromuscular performance of elite rugby union players and relationships with salivary hormones, Journal of Strength and Conditioning Research, 23 (2009) 2046-2053. https://doi.org/10.1519/jsc.0b013e3181b73c19 


\section{Acknowledgement}

NIL.

Informed consent

All participants gave written informed consents to participate in this study.

\section{Funding}

NIL

\section{Conflict of interest}

None of the authors have any conflicts of interest to declare.

\section{About The License}

(C) The author(s) 2020. The text of this article is open access and licensed under a Creative Commons Attribution 4.0 International License 